\title{
Correction to: Tightness for the cover time of the two dimensional sphere
}

\author{
David Belius ${ }^{1} \cdot$ Jay Rosen ${ }^{2} \cdot$ Ofer Zeitouni $^{3,4}$
}

Published online: 6 March 2020

(c) Springer-Verlag GmbH Germany, part of Springer Nature 2020

\section{Correction to: Probability Theory and Related Fields https://doi.org/10.1007/s00440-019-00940-2}

Lemma 9.1 in the paper is incorrect as stated. The proof given there yields the following corrected statement.

Lemma 9.1 For any $L-2 k \geq k^{\prime}>k+10 \geq 11$ and $n>1$, let $\mathcal{A}_{k^{\prime}}$ denote an event, measurable on the excursions of the Brownian motion from $\partial B_{d}\left(y, h_{k^{\prime}}\right)$ to $\partial B_{d}\left(y, h_{k^{\prime}-1}\right)$ during the first $n$ excursions from $\partial B_{d}\left(y, h_{k}\right) \rightarrow \partial B_{d}\left(y, h_{k-1}\right)$. Then,

$$
\begin{aligned}
& \mathbb{P}\left(\mathcal{A}_{k^{\prime}} \mid T_{y, k^{\prime}-1 \rightarrow k^{\prime}}^{y, \stackrel{n}{\rightarrow} k-1}=m_{k^{\prime}}, \mathcal{G}_{k}^{y}\right) \\
& \quad=\left(1+O\left(\left(k^{\prime}-k\right) \frac{h_{k^{\prime}-1}}{h_{k}}\right)\right)^{m_{k^{\prime}}} \mathbb{P}\left(\mathcal{A}_{k^{\prime}} \mid T_{y, k^{\prime}-1 \rightarrow k^{\prime}}^{y, k \stackrel{n}{\rightarrow} k-1}=m_{k^{\prime}}\right) .
\end{aligned}
$$

In particular, for all $m_{l} ; l=k^{\prime}, \ldots L$, and all $y \in \mathbf{S}^{2}$,

$$
\mathbb{P}\left(T_{y, l-1 \rightarrow l}^{y, k \stackrel{n}{\rightarrow} k-1}=m_{l} ; l=k^{\prime}+1, \ldots L \mid T_{y, k^{\prime}-1 \rightarrow k^{\prime}}^{y, k \stackrel{n}{\rightarrow} k-1}=m_{k^{\prime}}, \mathcal{G}_{k}^{y}\right)
$$

The original article can be found online at https://doi.org/10.1007/s00440-019-00940-2.

$\bowtie$ Ofer Zeitouni

ofer.zeitouni@weizmann.ac.il

David Belius

david.belius@cantab.net

Jay Rosen

jrosen30@optimum.net

1 Department of Mathematics and Computer Science, University of Basel, 4051 Basel, Switzerland

2 Department of Mathematics, College of Staten Island, CUNY, Staten Island, NY 10314, USA

3 Department of Mathematics, Weitzmann Institute, 76100 Rehovot, Israel

4 Department of Mathematics, Courant Institute, NYU, New York City, NY 10012, USA 


$$
\begin{aligned}
= & \left(1+O\left(\left(k^{\prime}-k\right) \frac{h_{k^{\prime}-1}}{h_{k}}\right)\right)^{m_{k^{\prime}}} \\
& \mathbb{P}\left(T_{y, l-1 \rightarrow l}^{y, k \stackrel{n}{\rightarrow} k-1}=m_{l} ; l=k^{\prime}+1, \ldots L \mid T_{y, k^{\prime}-1 \rightarrow k^{\prime}}^{y, k}=m_{k^{\prime}}\right) .
\end{aligned}
$$

In Lemma 9.1 we have $\left|O\left(\left(k^{\prime}-k\right) \frac{h_{k^{\prime}-1}}{h_{k}}\right)\right| \leq 100\left(\left(k^{\prime}-k\right) \frac{h_{k^{\prime}-1}}{h_{k}}\right)$.

This necessitates numerous corrections, mostly in the order in which estimates are applied. The changes occur in Section 4, as sketched below. Full details are given in the companion posting [2]. Here and in what follows, boldfaced numbers refer to the original numbering in [1].

1. Section 4.1 (first moment estimate) The estimate (4.56) is incorrect. Instead, one needs to use the decoupling estimates from Section 4.5. This necessitates changes starting from the paragraph following (4.47) through the end of the subsection, as follows.

Let

$$
\mathcal{W}_{y, k}^{\in x}(n)=\left\{d_{\mathrm{Wa}}^{1}\left(\frac{1}{n} \sum_{i=1}^{n} \delta_{\theta_{k, i}}, v_{k}\right) \in \frac{c_{0}}{2 \sqrt{n}} I_{x}\right\}
$$

so that

$$
\mathcal{W}_{y, k}^{c}\left(N_{k, a}\right) \subseteq \cup_{m=\log k}^{\infty} \mathcal{W}_{y, k}^{\in m}\left(N_{k, a}\right),
$$

and consequently, setting

$$
\mathcal{L}_{k, m, p, a}=\mathcal{K}_{k, p, a} \cap \mathcal{W}_{y, k}^{\in m}\left(N_{k, a}\right),
$$

we have

$$
\mathbb{P}\left(\mathcal{K}_{k, p, a}\right) \leq \sum_{m=\log k}^{\infty} \mathbb{P}\left(\mathcal{L}_{k, m, p, a}\right)
$$

Thus to prove (4.47) it suffices to show that for all $m \geq \log k$, all $d^{*} \leq k \leq L_{-}$, and all $0 \leq z \leq(\log L)^{1 / 4}$,

$$
\sum_{a=\left[k^{1 / 4}\right]}^{L^{3 / 4}} \sum_{p=\left[(k-3)^{1 / 4}\right]}^{L^{3 / 4}} \mathbb{P}\left(\mathcal{L}_{k, m, p, a}\right) \leq c(1+z) e^{-2 L} e^{-2 z} e^{-c^{\prime} m^{2}}
$$

Write

$$
\mathcal{L}_{k, m, p, a}^{\prime}=\mathcal{H}_{k-3, p} \bigcap \mathcal{H}_{k, a} \bigcap \mathcal{W}_{y, k}^{\in m}\left(N_{k, a}\right) .
$$

Since the $\theta_{k, i}$ are i.i.d. $v_{k}$-distributed random variables, as explained in the paragraph before (4.19), it follows from (4.17) that

$$
\mathbb{P}\left(\mathcal{W}_{y, k}^{\in m}\left(N_{k, a}\right) \mid T_{k-3}^{y, t_{z}}\right) \leq 2 e^{-m^{2} / 4}
$$

The following lemma is immediate, details in [2, Lemma 4.6]. 
Lemma 1.1 There exist constants $c, c^{\prime}>0$ so that

$$
\mathbb{P}\left(\mathcal{L}_{k, m, p, a}^{\prime}\right) \leq c e^{-2 k-2(z-p)-(z-p)^{2} / 4 k} e^{-m^{2} / 8} e^{-c^{\prime}(a-p)^{2}}
$$

Let

$$
\begin{gathered}
k^{+}=k+\left\lceil 10^{10} \log L\right\rceil, \quad k^{++}=k+2\left\lceil 10^{10} \log L\right\rceil, \\
\mathbb{B}_{y, k+3, k^{+}}^{j^{\prime}}=\left\{\gamma(l) \leq \sqrt{2 T_{l}^{y, t_{z}}} \text { for } l=k+3, \ldots, k^{+},\right. \\
\left.\sqrt{2 T_{k^{+}}^{y, t_{z}}} \in I_{\rho_{L}\left(L-k^{+}\right)+j^{\prime}}\right\},
\end{gathered}
$$

and

$$
\begin{gathered}
\widehat{\mathfrak{B}}_{y, k^{++}+1, L}=\left\{\rho_{L}(L-l) \leq \sqrt{2 T_{l}^{y, t_{z}}} \text { for } l=k^{++}+1, \ldots, L-1,\right. \\
\left.\sqrt{2 T_{L}^{y, t_{z}}}=0\right\} .
\end{gathered}
$$

We have that,

$$
\begin{aligned}
\mathbb{P}\left(\mathcal{L}_{k, m, p, a}\right)= & \mathbb{P}\left(\mathcal{L}_{k, m, p, a}^{\prime} \cap \mathcal{B}_{y, k+1, L}\right) \leq \sum_{j^{\prime}=\left(k^{+}\right)^{1 / 4}}^{L^{3 / 4}} \sum_{j^{\prime \prime}=\left(k^{++}\right)^{1 / 4}}^{L^{3 / 4}} \\
& \mathbb{P}\left(\mathcal{L}_{k, m, p, a}^{\prime} \cap \mathbb{B}_{y, k+3, k^{+}}^{j^{\prime}} \cap \widehat{\mathfrak{B}}_{y, k^{++}+1, L} ; \sqrt{2 T_{k^{++}}^{y, t_{z}}} \in I_{\rho_{L}\left(L-k^{++}\right)+j^{\prime \prime}}\right),
\end{aligned}
$$

up to an error due to the restriction of $j^{\prime}, j^{\prime \prime} \leq L^{3 / 4}$, which is little $o$ of the right-hand side of $[1,(4.43)]$. Let

$$
\begin{aligned}
& \mathcal{G}_{k^{+}}^{y} \\
& \quad=\sigma \text {-algebra generated by the excursions from } \partial B_{d}\left(y, h_{k^{+}-1}\right) \text { to } \partial B_{d}\left(y, h_{k^{+}}\right)
\end{aligned}
$$

(In the definition of $\mathcal{G}_{k^{+}}^{y}$, if we start outside $\partial B_{d}\left(y, h_{k^{+}-1}\right)$ we include the initial excursion to $\partial B_{d}\left(y, h_{k^{+}-1}\right)$. Do not confuse with (3.38).) Note that

$$
\mathcal{A}_{j^{\prime}}:=\mathcal{L}_{k, m, p, a}^{\prime} \cap \mathbb{B}_{y, k+3, k^{+}}^{j^{\prime}} \in \mathcal{G}_{k^{+}}^{y} .
$$

Using (1.14) we have

$$
\mathbb{P}\left(\mathcal{A}_{j^{\prime}} ; \sqrt{2 T_{k^{++}}^{y, t_{z}}} \in I_{\rho_{L}\left(L-k^{++}\right)+j^{\prime \prime}} ; \widehat{\mathfrak{B}}_{y, k^{++}+1, L}\right)
$$




$$
\begin{gathered}
\leq \sup _{s \in I_{\rho\left(L-k^{+}\right)+j^{\prime}}, v \in I_{\rho\left(L-k^{++}+j^{\prime \prime}\right.}} \mathbb{P}\left(\mathcal{A}_{j^{\prime}} ; \sqrt{2 T_{k^{++}}^{y, t_{z}}} \in I_{\rho\left(L-k^{++}\right)+j^{\prime \prime}} ;\right. \\
\left.\quad \mathbb{P}\left(\widehat{\mathfrak{B}}_{y, k^{++}+1, L} \mid T_{y, k^{++}-1 \rightarrow k^{++}}^{y, k^{s^{2} / 2} k^{+}-1}=v^{2} / 2, \mathcal{G}_{k^{+}}^{y}\right)\right) .
\end{gathered}
$$

By Lemma 9.1 with the $k, k^{\prime}$ there replaced by $k^{+}, k^{++}$and using that $\left(1+10\left(k^{++}-\right.\right.$ $\left.\left.k^{+}\right) h_{k^{++}} / h_{k^{+}}\right)^{4 L^{2}}$ is bounded above uniformly, we have that for some universal $c<\infty$

$$
\begin{aligned}
& \mathbb{P}\left(\widehat{\mathfrak{B}}_{y, k^{++}+1, L} \mid T_{y, k^{++}-1 \rightarrow k^{++}}^{y, k^{+} \stackrel{s^{2} / 2}{\rightarrow} k^{+}-1}=v^{2} / 2, \mathcal{G}_{k^{+}}^{y}\right) \\
& \leq c \mathbb{P}\left(\widehat{\mathfrak{B}}_{y, k^{++}+1, L} \mid T_{y, k^{++}-1 \rightarrow k^{++}}^{y, k^{s^{2} / 2} \rightarrow k^{+}-1}=v^{2} / 2\right) .
\end{aligned}
$$

By the barrier estimate of Lemma 8.4 in Appendix I, we see that for $j^{\prime \prime}$ in the range of summation in (1.12), uniformly in $s \in I_{\rho\left(L-k^{+}\right)+j^{\prime}}$ and $v \in I_{\rho\left(L-k^{++}\right)+j^{\prime \prime}}$,

$$
\mathbb{P}\left(\widehat{\mathfrak{B}}_{y, k^{++}+1, L} \mid T_{y, k^{++}-1 \rightarrow k^{++}}^{y, k^{s^{2} / 2} k^{+}-1}=v^{2} / 2\right) \leq c\left(1+j^{\prime \prime}\right) e^{-2\left(L-k^{++}\right)-2 j^{\prime \prime}} .
$$

Thus, uniformly in $\left[k^{1 / 4}\right] \leq a \leq L^{3 / 4}$,

$$
\begin{aligned}
\mathbb{P}\left(\mathcal{L}_{k, m, p, a}\right)= & \mathbb{P}\left(\mathcal{L}_{k, m, p, a}^{\prime} \cap \mathcal{B}_{y, k+3, L}\right) \leq c e^{-2\left(L-k^{++}\right)} \\
& \times \sum_{j^{\prime}=\left(k^{+}\right)^{1 / 4}}^{L^{3 / 4}} \sum_{j^{\prime \prime}=\left(k^{++}\right)^{1 / 4}}^{L^{3 / 4}} j^{\prime \prime} e^{-2 j^{\prime \prime}} \mathbb{P}\left(\mathcal{L}_{k, m, p, a}^{\prime} \cap \mathbb{B}_{y, k+3, k^{+}, k^{++}}^{j^{\prime}, j^{\prime \prime}}\right),
\end{aligned}
$$

where

$$
\begin{aligned}
& \mathbb{B}_{y, k+3, k^{+}, k^{++}}^{j^{\prime}, j^{\prime \prime}}=\left\{\gamma(l) \leq \sqrt{2 T_{l}^{y, t_{z}}} \text { for } l=k+3, \ldots, k^{+}, k^{++},\right. \\
& \left.\sqrt{2 T_{k^{+}}^{y, t_{z}}} \in I_{\rho_{L}\left(L-k^{+}\right)+j^{\prime}}, \sqrt{2 T_{k^{++}}^{y, t_{z}}} \in I_{\rho_{L}\left(L-k^{++}\right)+j^{\prime \prime}}\right\} .
\end{aligned}
$$

From this point, the estimates needed are similar to the argument in [1, Section 4.1], using the barrier estimates of Section $\mathbf{8}$ according to different values of $m$. The full details and the division to different cases appear in [2, Section 4.1].

Section 4.4 (Second moment estimate: early branching) The last inequality in (4.87) is incorrect. In order to fix that, one needs to change the events to which one applies decoupling, starting from below $\mathbf{( 4 . 8 3 )}$, as follows. 
Recall the events $\mathbb{B}_{y, k+3, k^{+}}^{j^{\prime}}$ and $\widehat{\mathfrak{B}}_{y, k^{++}+1, L}$, and the $\sigma$-algebra $\mathcal{G}_{k^{+}}^{y}$. We have that

$$
\begin{gathered}
\mathbb{P}\left(\mathcal{W}_{y, k}\left(N_{k, a}\right) \cap \mathcal{B}_{y, k+3, L} \cap \mathcal{H}_{k, a} \cap \mathcal{I}_{y^{\prime}, z}\right) \leq \sum_{j^{\prime}=\left(k^{+}\right)^{1 / 4}}^{L^{3 / 4}} \sum_{j^{\prime \prime}=\left(k^{++}\right)^{1 / 4}}^{L^{3 / 4}} \\
\mathbb{P}\left(\mathcal{W}_{y, k}\left(N_{k, a}\right) \cap \mathbb{B}_{y, k+3, k^{+}}^{j^{\prime}} \cap \widehat{\mathfrak{B}}_{y, k^{++}+1, L} \cap \mathcal{H}_{k, a} \cap \widehat{\mathcal{I}}_{y^{\prime}, k, z},\right. \\
\left.\sqrt{2 T_{k^{++}}^{y, t_{z}}} \in I_{\rho_{L}\left(L-k^{++}\right)+j^{\prime \prime}}\right)+o\left(e^{-a} E(k)\right),
\end{gathered}
$$

where the error term is coming from the restriction $j^{\prime}, j^{\prime \prime} \leq L^{3 / 4}$. Next, set

$$
\mathcal{A}_{j^{\prime}}:=\mathcal{W}_{y, k}\left(N_{k, a}\right) \bigcap \mathcal{H}_{k, a} \bigcap \widehat{\mathcal{I}}_{y^{\prime}, k, z} \cap \mathbb{B}_{y, k+3, k^{+}}^{j^{\prime}} \in \mathcal{G}_{k^{+}}^{y}
$$

Using (1.21) we have

$$
\begin{gathered}
\mathbb{P}\left(\mathcal{A}_{j^{\prime}} ; \sqrt{2 T_{k^{++}}^{y, t_{z}}} \in I_{\rho_{L}\left(L-k^{++}\right)+j^{\prime \prime}} ; \widehat{\mathfrak{B}}_{y, k^{++}+1, L}\right) \\
\leq \sup _{s \in I_{\rho\left(L-k^{+}\right)+j^{\prime}}, v \in I_{\rho\left(L-k^{++}\right)+j^{\prime \prime}}} \mathbb{P}\left(\mathcal{A}_{j^{\prime}} ; \sqrt{2 T_{k^{++}}^{y, t_{z}}} \in I_{\rho\left(L-k^{++}\right)+j^{\prime \prime}}\right. \\
\left.\mathbb{P}\left(\widehat{\mathfrak{B}}_{y, k^{++}+1, L} \mid T_{y, k^{++}-1 \rightarrow k^{++}}^{y, k^{s^{2} / 2} k^{+}-1}=v^{2} / 2, \mathcal{G}_{k^{+}}^{y}\right)\right)
\end{gathered}
$$

Recall the estimates (1.16) and (1.17). We then have that uniformly in $\left[k^{1 / 4}\right] \leq a \leq L^{3 / 4}$,

$$
\begin{aligned}
& \mathbb{P}\left(\mathcal{W}_{y, k}\left(N_{k, a}\right) \cap \mathcal{B}_{y, k+3, L} \cap \mathcal{H}_{k, a} \cap \mathcal{I}_{y^{\prime}, z}\right) \\
& \leq c e^{-2\left(L-k^{++}\right)} \sum_{j^{\prime}=\left(k^{+}\right)^{1 / 4}}^{L^{3 / 4}} \sum_{j^{\prime \prime}=\left(k^{++}\right)^{1 / 4}}^{L^{3 / 4}}\left(1+j^{\prime \prime}\right) e^{-2 j^{\prime \prime}} \\
& \quad \times \mathbb{P}\left(\mathcal{W}_{y, k}\left(N_{k, a}\right) \cap \mathbb{B}_{y, k+3, k^{+}, k^{++}}^{j^{\prime}, j^{\prime \prime}} \cap \mathcal{H}_{k, a} \cap \widehat{\mathcal{I}}_{y^{\prime}, k, z}\right),
\end{aligned}
$$

where $\mathbb{B}_{y, k+3, k^{+}, k^{++}}^{j^{\prime}, j^{\prime \prime}}$ is as in (1.19).

The next lemma handles the last term in (1.23) and replaces Lemma 4.7 in [1]. The proof, which is given in detail in [2, Lemma 4.9], is very similar to the proof of Lemma 4.7; it uses the decoupling lemma (Lemma 4.11), barrier estimates and the control on Wasserstein distance contained in (4.17).

Lemma 1.2 For some $M_{0}<\infty$ and $k, a, j^{\prime}$ in the ranges specified above, 


$$
\begin{aligned}
& \mathbb{P}\left(\mathcal{W}_{y, k}\left(N_{k, a}\right) \bigcap \mathbb{B}_{y, k+3, k^{+}, k^{++}}^{j^{\prime}, j^{\prime \prime}} \bigcap \mathcal{H}_{k, a} \bigcap \widehat{\mathcal{I}}_{y^{\prime}, k, z}\right) \\
& \quad \leq F_{k, a, j^{\prime}, j^{\prime \prime}} \mathbb{P}\left(\widehat{\mathcal{I}}_{y^{\prime}, k, z}\right)+e^{-2 L-\sqrt{L}}
\end{aligned}
$$

where

$$
\begin{aligned}
& F_{k, a, j^{\prime}, j^{\prime \prime}=} c a e^{-2 a} e^{-2\left(k^{++}-k\right)} \sum_{\substack{\left\{\bar{j}^{\prime}:\left|\bar{j}^{\prime}-j^{\prime}\right| \leq 2 M_{0} \log k\right\} \\
\left\{\bar{j}^{\prime \prime}:\left|\bar{j}^{\prime \prime}-\bar{j}^{\prime \prime}\right| \leq 2 M_{0} \log k\right\}}} \\
& \bar{j}^{\prime} \frac{e^{-\left(\bar{j}^{\prime}-a\right)^{2} /\left(4\left(k^{+}-k\right)\right)}}{\left(k^{+}-k\right)} e^{2 \bar{j}^{\prime \prime}} \frac{e^{-\left(\bar{j}^{\prime}-\bar{j}^{\prime \prime}\right)^{2} / 2\left(k^{+}-k\right)}}{\left(k^{+}-k\right)} .
\end{aligned}
$$

Assuming Lemma 1.2, the rest of the proof is similar to [1, Section 4.4]. Full details appear in [2, Section 4.4].

\section{References}

1. Belius, D., Rosen, J., Zeitouni, O.: Tightness for the cover time of the two dimensional sphere. Probab. Theory. Relat. Fields 176, 1357-1437 (2020)

2. Belius, D., Rosen, J., Zeitouni, O.: Tightness for the cover time of the two dimensional sphere. (2020). arXiv: $1711.02845 \mathrm{v} 6$

Publisher's Note Springer Nature remains neutral with regard to jurisdictional claims in published maps and institutional affiliations. 